\title{
Metro Add-Drop Network Applications of Cascaded Dispersion-Compensated Interleaver Pairs Using a Recirculating Loop
}

\author{
Kai-Ming Feng, Member, IEEE, Ming-Fang Huang, Chia-Chien Wei, Chung-Yu Lai, Tse-Yu Lin, \\ Jason (Jyehong) Chen, Member, IEEE, and Sien Chi
}

\begin{abstract}
A 50-GHz channel spaced dispersion-compensated interleaver pair for a metro add-drop network application was demonstrated using a recirculating loop. After five cascaded nodes (ten interleavers), a 2.5-dB sensitivity differential at bit-error-rate level around $10^{-9}$ was observed between the compensated and uncompensated pairs within a $\pm 10-\mathbf{G H z}$ detuning frequency window.
\end{abstract}

Index Terms-Interleaver pair, optical fiber communication, optical fiber device, recirculating loop.

\section{INTRODUCTION}

I N RECENT years, research on wavelength-division-multiplexing (WDM) systems has dramatically increased [1]. The reconfigurability of WDM networks has the following features: The network's configuration can be increased by bypassing, adding, or dropping the traffic and the capacity throughput enlarges when the traffic is multiplexed on the fiber by WDM. Many dense wavelength-division-multiplexing (DWDM) systems employ interleavers as multiplexers and demultiplexers for combining or separating even and odd channels [1]-[3]. In a metro scheme, a pair of interleavers is utilized in add-drop applications to provide up to $50 \%$ adding and dropping of the total traffic while simultaneously reducing the insertion loss associated with the express channels. In such an application, the two factors that restrict the maximum number of cascadable nodes are the passbands' flatness (amplitude response) and group delay (phase response) [1]-[5]. As the data rate increases, the system becomes more sensitive to the dispersion variations within the signal bandwidth. Accordingly, the flattened phase response is a crucial parameter in determining the usable bandwidth of the passband in 40-Gb/s systems [6], [7].

This investigation presents a dispersion-compensated interleaver pair in a cascadability study using a recirculating loop. The interleavers had a $0.5-\mathrm{dB}$ passband that exceeded

Manuscript received November 22, 2004; revised February 3, 2005. This work was supported by the National Science Council of the Republic of China, Taiwan, under Contract NSC 93-2215-E-009-027, Contract NSC 93-2219-E-007-001, Contract NSC 93-2752-E009-009-PAE, Contract NSC 93-2215-E-115-004, Contract NSC 93-2215-E-115-005, and Contract NSC 93-2752-E-007-002-PAE

K.-M. Feng is with the Institute of Communication Engineering, National Tsing Hua University, Hsin-Chu 300, Taiwan, R.O.C.

M.-F. Huang, C.-C. Wei, C.-Y. Lai, T.-Y. Lin, J. Chen, and S. Chi are with the Institute of Electro-Optical Engineering and Department of Photonics, National Chiao-Tung University, Hsin-Chu 300, Taiwan, R.O.C. (e-mail: kmfeng@ com.nthu.edu.tw).

Digital Object Identifier 10.1109/LPT.2005.846463
$35 \mathrm{GHz}$, and they supported both the positive and negative phase responses. The channel spacing of an interleaver pair was $50 \mathrm{GHz}$ for multiplexing-demultiplexing odd and even channels in a DWDM system. When the wavelength was detuned by $\pm 10 \mathrm{GHz}$ for the uncompensated pairs, a 2.5 -dB receiving sensitivity differential was observed after five cascaded nodes (ten interleavers), while the sensitivity differential is negligible for the compensated pairs.

\section{DeVICE Characteristic}

The interleaver designed and fabricated in this work is a symmetrical four-port interleaver with two input and two output ports. The details of the interleaver technologies, including principle of operation, architectures, and design rules can be found in [3] and [6]. Fig. 1(a) shows two possible connections for such an interleaver. For Type I connection, the odd and even channels experience a convex group delay characteristic, while channels experience a concave group delay for Type II connection. Fig. 1(b) illustrates the measured corresponding group delay curves for both connections in an interleaver's passband. These two types of connections with two mirrored group delay can be cascaded to generate a linear phase interleaver pair that the total dispersion would be near zero. Fig. 1(c) presents the group delay curves for the compensated and uncompensated interleaver pair connections. Clearly, for the compensated connection, the induced in-band dispersion is insignificant. However, when the interleaver pair is connected without compensation, the dispersion, induced by each individual interleaver, accumulates. If such interleaver pairs are cascaded in a metro add-drop network, the interleaver-induced dispersion aggregates and limits the maximum cascadable nodes. Fig. 1(d) displays the measured group delay curves accumulated after five cascaded interleaver pairs for the connections with and without dispersion compensation. The experimental results show that the wavelengths should precisely coincide with the ITU grids for the uncompensated case, otherwise a significant interleaver-induced dispersion accumulates. However, no noticeable interleaver-induced dispersion was built up after five add-drops for the compensated case.

\section{CASCADING ADD-Drops SYSTEM EXPERIMENT}

To demonstrate the feasibility of cascading interleaver pairs for add-drop application in metro networks, a recirculating loop was employed to simulate multiple add-drops in a ring network. Fig. 2 shows the experimental setup of the recirculating loop. 


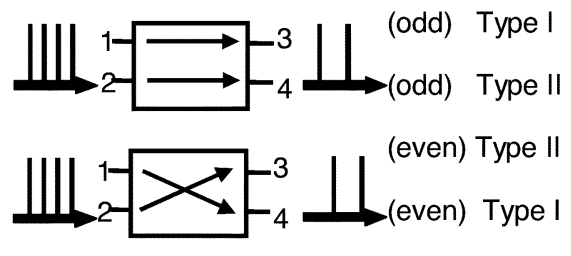

(a)

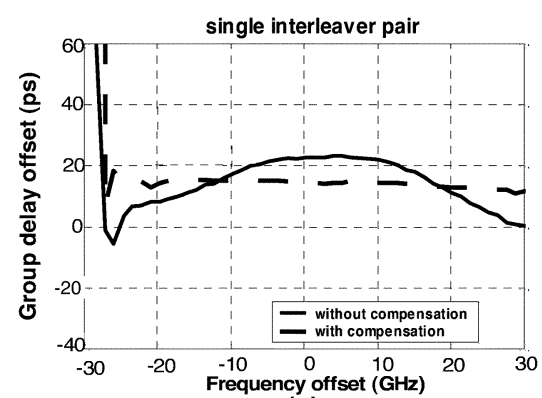

(c)

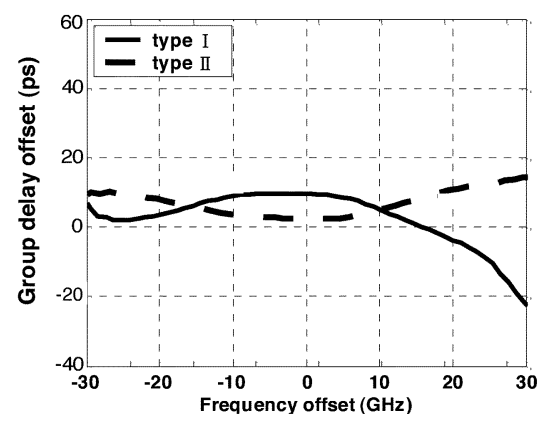

(b)

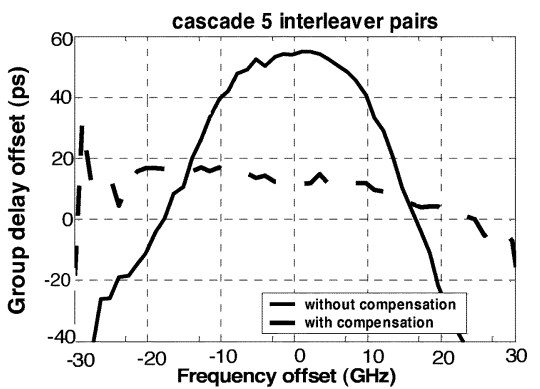

(d)

Fig. 1. (a) Two operation connections for a four-port interleaver. (b) In-band group delays for two types of interleaver connection. (c) Group delays of compensated and uncompensated interleaver-pair connections. (d) Group delays of two cases after five cascaded interleaver pairs.

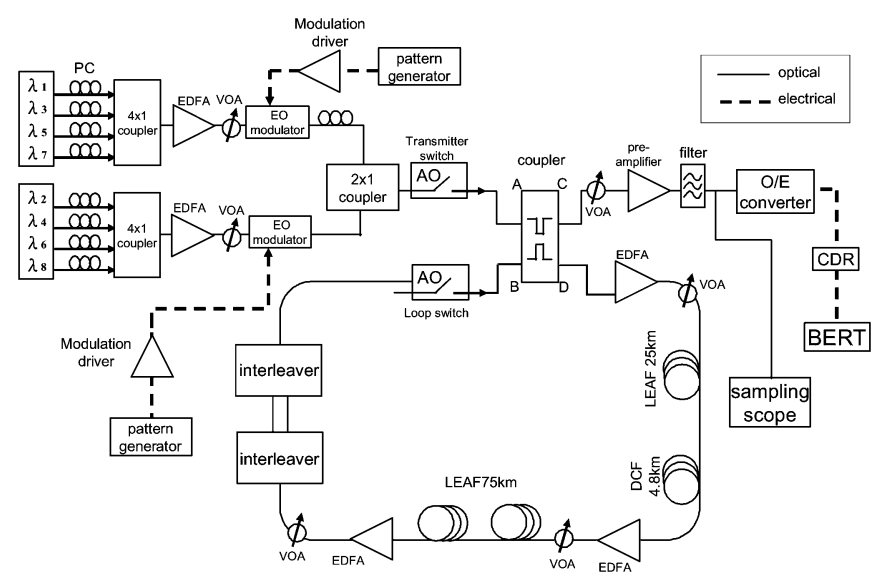

Fig. 2. Experimental setup of a recirculating loop.

The eight channel laser sources consisted of two groups: one from 193.2 to $193.35 \mathrm{THz}$ and the other from 192.7 to $192.85 \mathrm{THz}$ all with $50-\mathrm{GHz}$ channel spacing. The odd and even channels were individually modulated by a $\mathrm{LiNbO}_{3}$ electrooptical modulator at $10 \mathrm{~Gb} / \mathrm{s}$ with a pseudorandom binary sequence length of $2^{31}-1$ patterns. A polarization controller was employed to set the polarization state of the odd channels to be orthogonal to that of the even channels to reduce the deleterious nonlinear effects. Two types of fiber were used in the recirculating loop: $100 \mathrm{~km}$ of Corning LEAF fiber and $4.8 \mathrm{~km}$ of Corning DCF, with $-86.6231-\mathrm{ps} / \mathrm{nm} / \mathrm{km}$ dispersion at $193.0 \mathrm{THz}$, to compensate the accumulated chromatic dispersion in LEAF fiber. The fully compensated wavelength of this fiber loop was located at around $193.15 \mathrm{THz}$. After $105 \mathrm{~km}$ of fiber, the interleaver pair was inserted in the fiber loop to simulate optical channel add-drop at every $105 \mathrm{~km}$. A 3R receiver of $-33-\mathrm{dBm}$ back-to-back sensitivity at bit-error-rate (BER)

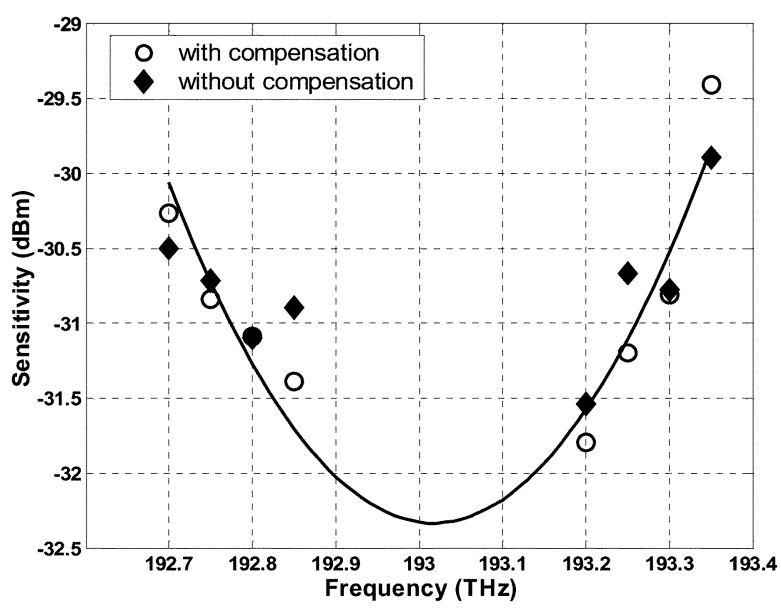

Fig. 3. Receiving sensitivity variation of compensated and uncompensated cases after five cascaded interleaver pairs.

equal to $10^{-9}$ was applied to obtain the signal's performance after transmission.

Fig. 3 compares the receiving sensitivity at BER level around $10^{-9}$ of each channel after five loops, i.e., $525 \mathrm{~km}$ of transmission and five add-drop nodes, for both compensated and uncompensated cases. Since the two groups of channels were deliberately selected to locate at the wavelength regions with opposite sign of dispersion: one group with negative dispersion, and the other one with positive dispersion, the experimental results for both cases indicate that the receiving sensitivities of eight- channels exhibit a parabolic distribution centered at dispersion-zero wavelength. The sensitivity difference for each channel is insignificant between the two cases because, as the channel wavelengths are set at the center of the interleaver's passband precisely, very little dispersion is introduced by the 


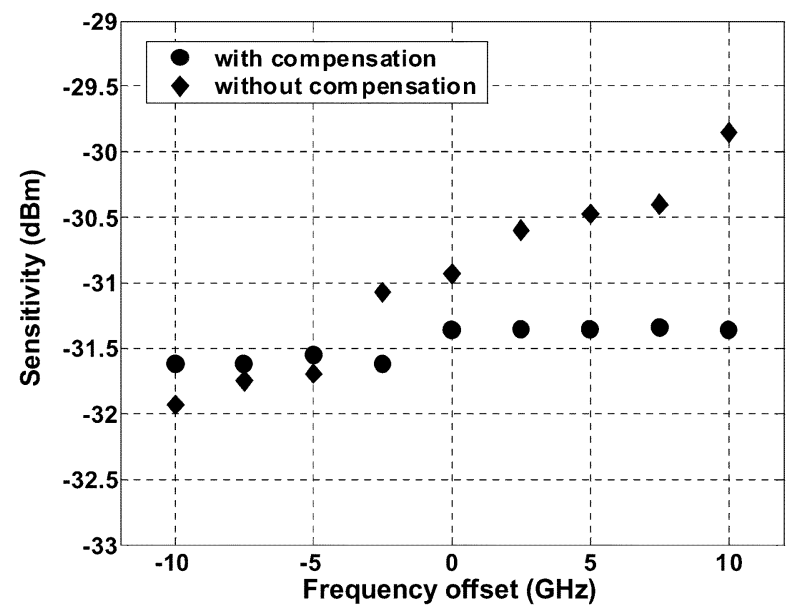

Fig. 4. Receiving sensitivity variation at $\mathrm{BER}=10^{-9}$ for both compensated and uncompensated configuration with $\pm 10-\mathrm{GHz}$ wavelength detuning.

interleaver, even for the uncompensated case. Thus, the parabolic sensitivity distribution of the eight channels for both cases mainly results from the residual dispersion accumulation after $525 \mathrm{~km}$.

Fig. 4 compares the receiver sensitivity variations, with $\pm 10-\mathrm{GHz}$ wavelength detuning, of the compensated and uncompensated configurations. This figure indicates that less than $0.4-\mathrm{dB}$ sensitivity variation is observed within $\pm 10-\mathrm{GHz}$ wavelength detuning for the compensated connection, while more than 2.5-dB sensitive variation is perceived for the uncompensated case at BER $=10^{-9}$. Fig. 5 shows the BER curves and the corresponding eye diagrams when the wavelength is either on the ITU grid or detuned from the ITU wavelength by $\pm 8 \mathrm{GHz}$ at Channel 5. Both the eye diagrams and the BER curves indicate that the accumulated dispersion in the uncompensated connection will lead to pulse distortion and BER pentalty. Fig. 5(a) shows that the sensitivity was improved more than $2.5 \mathrm{~dB}$ when the wavelength detuned $\pm 8 \mathrm{GHz}$ from the center wavelwngth. It is because the dispersion from transmission fiber and the interleaver pairs are opposite and, thus, compensated each other when wavelength is detunned by $-8 \mathrm{GHz}$. However, the residue dispersion from fiber and interleaver pairs add up and cause the pulse distortion, as shown in the inset, when the wavelength detuned $+8 \mathrm{GHz}$. Conversely, if the interleaver pair is in the dispersion compensation connection, wavelength detuning up to $\pm 8 \mathrm{GHz}$ does not introduce much sensitivity variation. Consequently, the dispersion compensated connection is less sensitive than uncompensated one to the wavelength deviation from ITU grids.

\section{CONCLUSION}

This investigation presents a cascadability study of a $50-\mathrm{GHz}$ channel spacing dispersion-compensated interleaver pair using a recirculating loop. After five cascaded nodes and $525 \mathrm{~km}$ of transmission, the interleaver-induced dispersion did not significantly degrade the system performance when wavelengths were exactly aligned to ITU grids. However, when the wavelength deviated from ITU grids, the sensitivity variation of the compensated case was much less than that of the uncompensated pair.

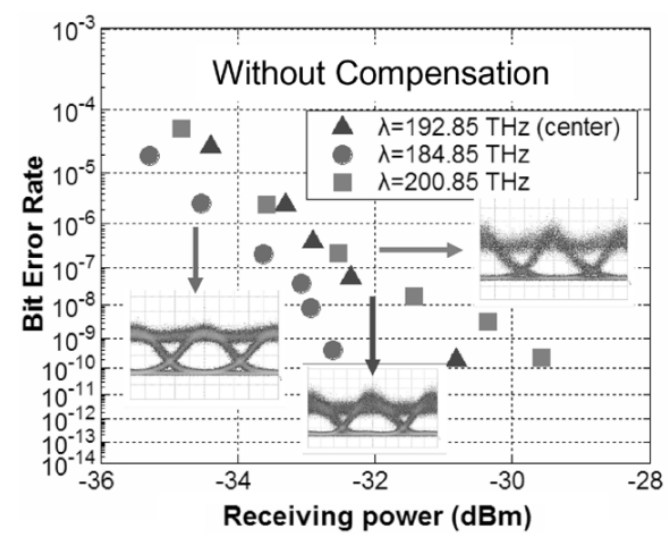

(a)

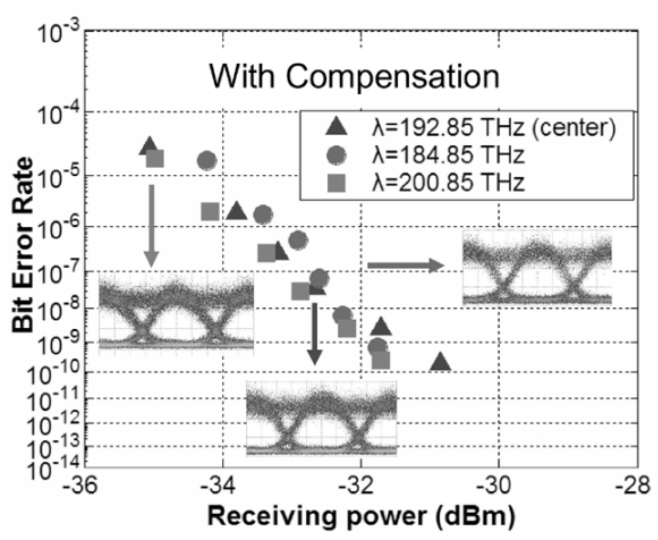

(b)

Fig. 5. BER curves and corresponding eye diagrams at channel five when wavelength is detuned for $\pm 8 \mathrm{GHz}$ (a) with compensation and (b) without compensation.

Experimental results show that, with $\pm 10-\mathrm{GHz}$ wavelength detuning, the sensitivity variations of these two configurations are less than $0.4 \mathrm{~dB}$ and more than $2.5 \mathrm{~dB}$, for compensated and uncompensated cases, respectively. Such results can lessen the precision requirements in selecting DFB lasers for metro add-drop network applications.

\section{REFERENCES}

[1] S. Y. Kim, S. H. Lee, S. S. Lee, and J. S. Lee, "Upgrading WDM networks using ultradense WDM channel groups," IEEE Photon. Technol. Lett., vol. 16, no. 8, pp. 1966-1968, Aug. 2004.

[2] E. Almstrom, S. N. Larsson, and H. Carlden, "Cascadability of optical add/drop multiplexers," in Proc. 24th Eur. Conf., vol. 1, 1998, pp. 589-590.

[3] S. Cao, J. Chen, J. N. Damask, C. R. Doerr, L. Guiziou, G. Harvey, Y. Hibino, H. Li, S. Suzuki, K.-Y. Wu, and P. Xie, "Interleaver technology: Comparisons and applications requirements," J. Lightw. Technol., vol. 22, no. 1, pp. 281-289, Jan. 2004.

[4] G. Lenz, B. J. Eggleton, C. R. Giles, C. K. Madsen, and R. E. Slusher, "Dispersive properties of optical filters for WDM systems," IEEE $J$. Quantum Electron., vol. 34, no. 8, pp. 1390-1402, Aug. 1998.

[5] A. Zeng, X. Ye, J. Chon, and F. Liang, " $25 \mathrm{GHz}$ interleavers with ultra-low chromatic dispersion," in Optical Fiber Communication Conf., 2002, pp. 369-398.

[6] J. Chen, "Dispersion-compensating optical digital filters for $40-\mathrm{Gb} / \mathrm{s}$ metro add-drop applications," IEEE Photon. Technol. Lett., vol. 16, no. 5, pp. 1310-1312, May 2004.

[7] K. Tai, Q. Guo, K. Chang, and J. Chen, "4-port interleavers and fully circulating bi-directional circulators," in Optical Fiber Communication Conf. 2001, 2001, pp. MK5/1-MK5/4. 